\title{
Abnormality of Gamete Formation in a Pummelo [Citrus maxima (Burm.) Merr.] Haploid
}

\author{
Masaki Yahata ${ }^{1,2 * *}$, Hisato Kunitake ${ }^{1}$, Kiichi Yasuda ${ }^{1}$, Takanori Hirai ${ }^{3}$, Tsutomu Yabuya ${ }^{1}$, \\ Kensuke Yamashita ${ }^{1}$ and Haruki Komatsu ${ }^{3 *}$
}

${ }^{1}$ Faculty of Agriculture, University of Miyazaki, Miyazaki 889-2192, Japan

${ }^{2}$ The United Graduate School of Agricultural Sciences, Kagoshima University, Kagoshima 890-0065, Japan

${ }^{3}$ School of Agriculture, Tokai University, Kumamoto 869-1404, Japan

\begin{abstract}
We investigated female and male gamete formation in a pummelo [Citrus maxima (Burm.) Merr.] haploid. During female gamete formation, no embryo-sac mother cell (EMC) was formed in ovules, and consequently no normal embryo sac was observed at anthesis, which was confirmed as the cause of female sterility in the haploid. During male gamete formation, on the other hand, meiosis in the pollen mother cells (PMCs) of the haploid occurred twice, but abnormalities were observed in most dividing cells. In some dividing cells, univalents did not distribute to the opposite poles at anaphase I; instead, they remained near the equatorial plate. The nine univalents then performed mitosis, which distributed equally forward opposite poles. Furthermore, at the tetrad stage, the tetrad comprised $99.3 \%$ of the 'Banpeiyu' pummelo, whereas sporads from monads to hexads with a relatively high frequency of dyads $(24.7 \%)$ were produced in the haploid. These results suggest that fertile pollen grains observed in the haploid pummelo were produced by abnormalities in the first meiotic division such as first division restitution (FDR).
\end{abstract}

Key Words: first division restitution (FDR), histological study, megasporogenesis, meiosis, microsporogenesis, unreduced gamete.

\section{Introduction}

Haploids have only one set of chromosomes, so no homologous chromosomes are available for synapsis. In the meiosis of haploids, only univalents appear at metaphase I, and their chromosomes separate unequally to the opposite poles at anaphase I. Haploids thus show sterility even if meiosis occurs.

However, some species of haploid plants can form fertile gametes (Veilleux, 1985; Yan et al., 2000). In fruit crops, this phenomenon has been observed only in Prunus persica Batsch (Hesse, 1971; Pooler and Scorza, 1995). Regarding the mechanism of fertility restitution,

Received; March 25, 2010. Accepted; July 21, 2010.

This research was supported by Diet and Cancer Prevention: Exploring Research Technology, Miyazaki Prefecture Collaboration of Regional Entities for the Advancement of Technological Excellence, JST, and the Sasakawa Scientific Research Grant from The Japan Science Society.

* Corresponding author (E-mail: hkomatsu@agri.u-tokai.ac.jp).

** Present address: Faculty of Agriculture, Shizuoka University, Shizuoka 422-8529, Japan.
Pooler and Scorza (1995) noted that all chromosomes in the meiocyte migrated to the same pole by chance during meiosis I, resulting in the formation of fertile unreduced gametes, whereas Hesse (1971) suggested that parallel spindle formation during meiosis resulted in a second division restitution. In the haploid plant of Capsicum annum L., Yan et al. (2000) found laggards in many dividing cells of the first division at meiosis, which resulted in first division restitution (FDR) at meiosis that led to the restitution of fertility in the haploid.

In Citrus and related genera, some haploid plants have been produced (Aleza et al., 2009; Froelicher et al., 2007; Germanà, 2009; Germanà and Chiancone, 2001, 2003; Germanà et al., 1994; Hidaka et al., 1979; Oiyama and Kobayashi, 1993; Toolapong et al., 1996; Yahata et al., 2010), but flowering of haploids has rarely been reported in these studies because the haploids were very weak and grew more slowly than the original diploid plants. To date, the only flowering haploids in Citrus have been a pummelo haploid from among small seed-derived seedlings obtained from the cross between 'Banpeiyu' 
pummelo and 'Ruby Red' grapefruit (C.paradisi Macfad.) (Toolapong et al., 1996) and a 'Clemenules' Clementine (C. clementina hort. ex Tanaka) haploid by gynogenesis in situ, induced by irradiated pollen (Aleza et al., 2009). Notably, the pummelo haploid produced by Toolapong et al. (1996) showed vigorous growth and flowered for the first time seven years after germination when it was grafted onto a trifoliate orange. Yahata et al. (2005a) reported that some diploid seeds were obtained when the pummelo haploid was crossed as the pollen parent with diploid cultivars, whereas reverse crosses resulted in no fruit set. However, the detailed process of female and male gamete formation in the pummelo haploid has not been completely clarified.

In the present study, we performed detailed cytogenetic analysis of female and male gamete formation in a haploid plant derived from a seedling of 'Banpeiyu' pummelo (Toolapong et al., 1996).

\section{Materials and Methods}

\section{Plant materials}

A pummelo haploid obtained from the cross between 'Banpeiyu' pummelo and 'Ruby Red' grapefruit (Toolapong et al., 1996) was used in the present study. The haploid had been grafted onto a trifoliate orange (Poncirus trifoliata (L.) Raf.), and maintained for approximately 10 years in the greenhouse of the School of Agriculture, Tokai University, Japan. An approximately 10-year-old 'Banpeiyu' pummelo grown in the orchard of Mr. Kiyoto Harusaki (Yatsusiro, Kumamoto, Japan) was used as the control.

\section{Meiotic behavior during megasporogenesis}

Flowers from 2 days after flowering (DAF) and flower buds of different developmental stages, i.e., 4/5, 3/4, $2 / 3,1 / 2,2 / 5,1 / 3,1 / 4$, and $1 / 5$ size of flower buds (SOFB) were collected from the haploid and 'Banpeiyu' pummelo and used to observe the female gamete. Sampled flowers and flower buds were fixed in FAA solution (formalin: glacial acetic acid: 70\% ethanol, $5: 5: 90 \mathrm{v} / \mathrm{v} / \mathrm{v})$ immediately after collection. After fixation, the petals, stamens, sepals, and style were removed from each sample, and the ovaries were dehydrated in a graded n-butyl alcohol series, and embedded in paraffin. After embedding, serial longitudinal sections were cut at $15 \mu \mathrm{m}$ with a rotary microtome (Nippon Optical Works, Tokyo, Japan) and stained with safranin and fast green. The meiotic process in the ovules was observed under a light microscope (BX51, Olympus, Tokyo, Japan) using these sections. At least 80 ovules were observed for each genotype and stage.

\section{Meiotic behavior during microsporogenesis and pollen fertility}

Cytogenetic analysis of the process of male gamete formation was performed according to the method of Yamashita and Yamaguchi (1992) with some modifica- tions. Flower buds of the haploid and the 'Banpeiyu' pummelo from $1 / 5$ to $1 / 4$ SOFB were collected and fixed in FAA solution. After fixation, squash preparations were made in a $1 \mathrm{mg} \cdot \mathrm{L}^{-1}$ 4'-6-diamidino-2-phenyl-indole (DAPI) solution on a slide, and meiosis of the pollen mother cell (PMC) was observed under a fluorescence microscope with a UV filter cassette. At the tetrad stage, meiocytes derived from PMC per genotype were counted.

Five stamens were collected, each from different flowers in the haploid and the 'Banpeiyu' pummelo, and were used for testing the stainability of the pollen grains. Pollen stainability was estimated by staining the samples with $1 \%$ acetocarmine after squashing nearly mature anthers on a slide glass. At least 1000 pollen grains were observed for each genotype with three repetitions.

\section{Results and Discussion}

\section{Meiotic behavior during megasporogenesis}

Formation of embryo-sac mother cells (EMCs) was first recognized in the ovules at $1 / 4 \mathrm{SOFB}$ of the 'Banpeiyu' pummelo (Figs. 1 and 2A). Subsequently, initiation of meiosis and tetrad formation were observed at 1/3 SOFB and 2/5 SOFB, respectively (Fig. 2B, C). Approximately $20 \%$ of the ovules contained EMCs or further developed embryo sacs. Embryo sacs developed rapidly thereafter during the flowering stage (Fig. 2D, E, F), and embryo sacs at the two-nucleate stage were observed at 2/3 SOFB. Eight-nucleate mature embryo sacs were formed in the flowers of 2 DAF (Fig. 2G), at a frequency of approximately $25 \%$. In the haploid, on the other hand, no EMCs were formed during flower bud development, and no embryo sac was formed in the flowers of 2 DAF (Fig. 1). Although the 'Banpeiyu' pummelo had normal morphologies in the inner and outer integuments, the haploid showed abnormalities in its ovules such as detached growth of integuments from nucellar tissue (Fig. 3A), and the formation of a void between the inner and outer integument (Fig. 3B).

In Citrus species, the formation of embryo sacs is incomplete at the flowering stage, and the sacs remain at the two- or four-nucleate stage until the mature embryo sacs are formed at 3 or 4 DAF (Bacchi, 1943; Iwamasa, 1976). In the present study, the 'Banpeiyu' pummelo showed normal meiosis and produced mature embryo sacs at 2 DAF although the formation frequency was low, whereas in the haploid the female gamete formation was abnormal and no EMC was formed the ovules. Furthermore, the morphologies of the inner and outer integuments of ovules in the pummelo haploid were similar to those of the haploid plant of 'Clemenules' clementine (Aleza et al., 2009).

\section{Meiotic behavior during microsporogenesis}

The male meiosis of the 'Banpeiyu' pummelo occurred normally. In the first meiotic division at prophase I, duplicated chromatin condensed (Fig. 4A), 
$\square$ Undifferentiated

- Tetrad

Four-nucleate embryo sac $\square$ Embryo-sac mother cell

$\square$ One-nucleate embryo sac

- Eight-nucleatei embryo sac $\square$ Meiosis

$\otimes$ Two-nucleate embryo sac

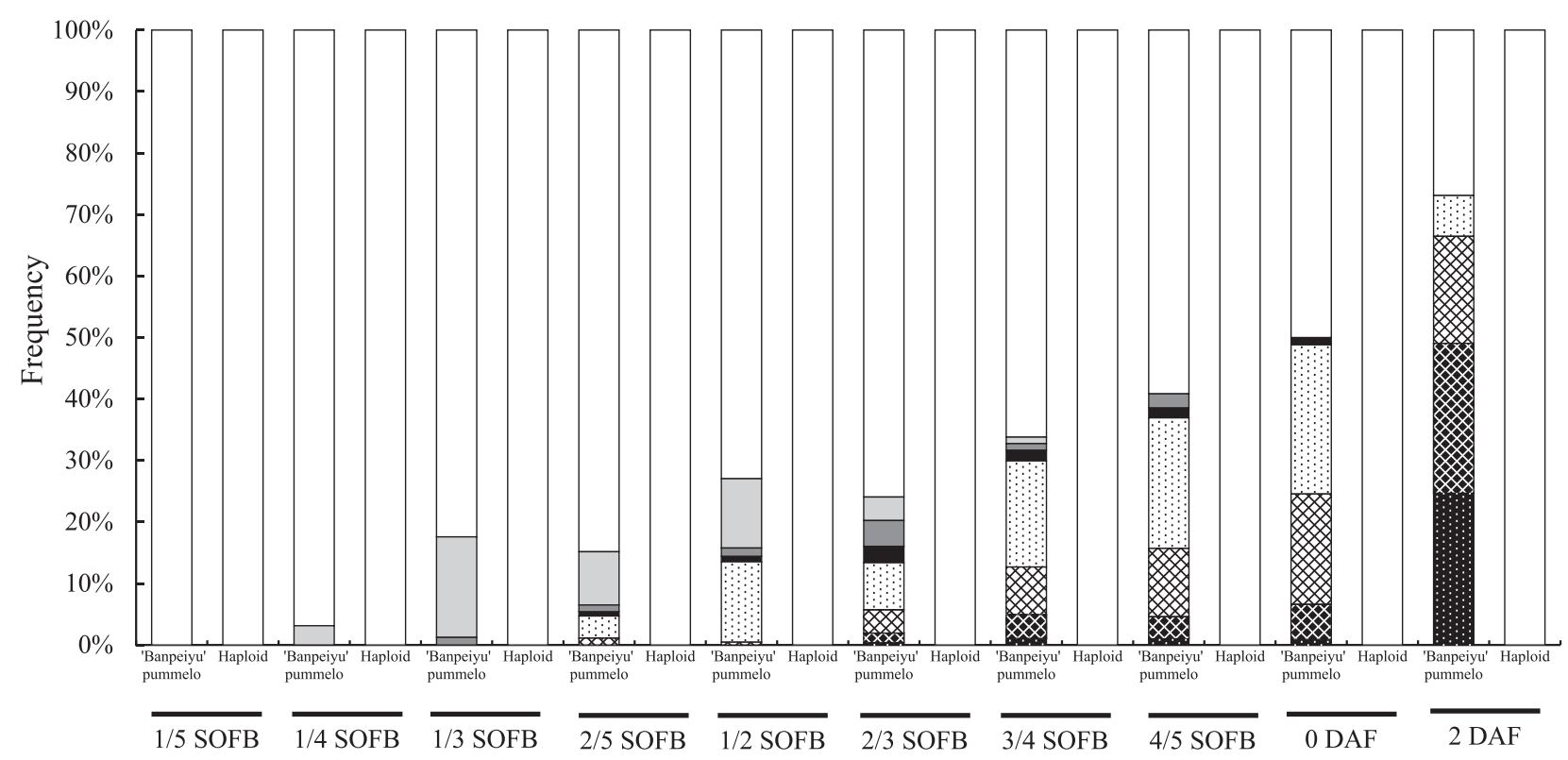

Stage

Fig. 1. Comparison of the developmental process of the embryo sac between the haploid and the 'Banpeiyu' pummelo from $1 / 5$ size flower buds (SOFB) to 2 days after flowering (DAF).

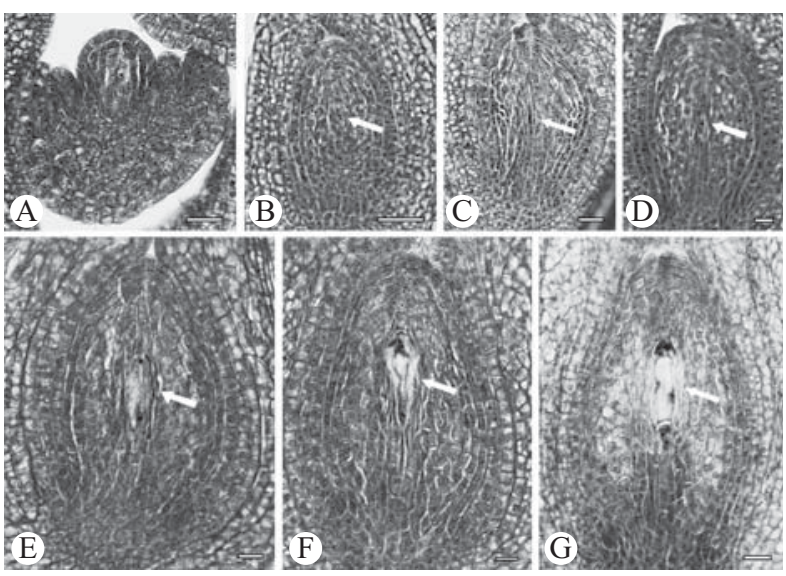

Fig. 2. Developmental stage of the embryo sac in the 'Banpeiyu' pummelo from $1 / 5$ size flower buds (SOFB) to 2 days after flowering (DAF). Arrows indicate the embryo sac: (A) differentiation of EMC at 1/4 SOFB; (B) meiosis at 1/3 SOFB; (C) tetrad stage at $2 / 5 \mathrm{SOFB}$; (D) one-nucleate embryo sac at 1/2 SOFB; (E) two-nucleate embryo sac at 3/4 SOFB; (F) fournucleate embryo sac at $0 \mathrm{DAF}$; and $(\mathrm{G})$ eight-nucleate embryo $\mathrm{sac}$ at $2 \mathrm{DAF}$. Scale bars $=10 \mu \mathrm{m}$.

and condensed chromosomes were visible. At metaphase I, homologous chromosomes aligned at the equatorial plate, and 9 bivalents were observed (Fig. 4B). The bivalents separated into univalents and migrated towards each pole at anaphase I (Fig. 4C, D). In the second division, chromosomes aligned at the equatorial plate at

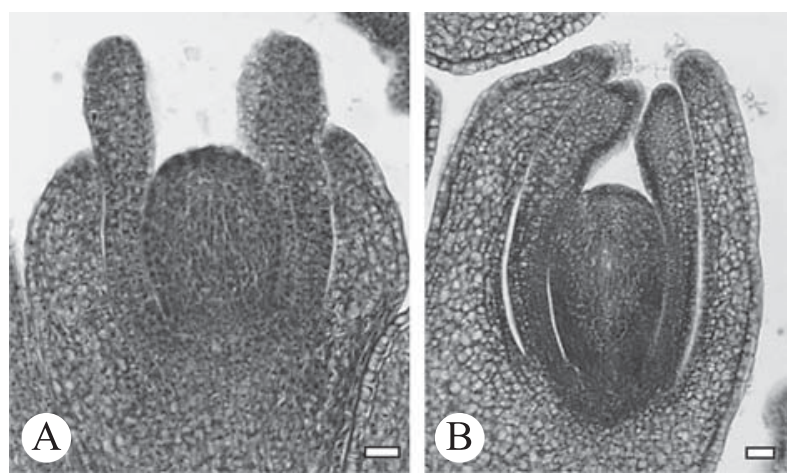

Fig. 3. Abnormal ovule development of the haploid: (A) the detached growth of integuments from nucellar tissue at $2 / 3$ size of flower buds (SOFB); and (B) the formation of a void between the inner and outer integument at $3 / 4$ SOFB. Scale bars $=20 \mu \mathrm{m}$.

metaphase II (Fig. 4E) and chromatids migrated towards each pole at anaphase II (Fig. 4F, G). Consequently, the 'Banpeiyu' pummelo produced a tetrad having four microspores of equal size (Fig. 4H).

In the pummelo haploid, on the other hand, meiotic division occurred twice in the PMC, but abnormalities were observed in most dividing cells (Fig. 5). Although nine univalents aligned on the equatorial plate at metaphase I (Fig. 5A, B), they migrated unequally to each pole (Fig. 5C, D). In the second division, their chromatids also migrated separately to each pole 


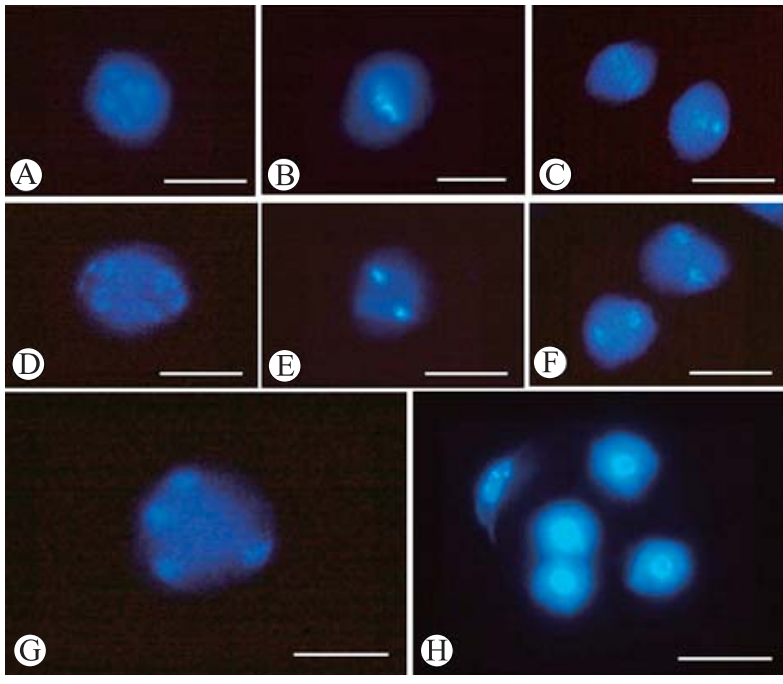

Fig. 4. Meiotic stages of the 'Banpeiyu' pummelo: (A) prophase I; (B) metaphase I; (C) anaphase I; (D) telophase I and prophase II; (E) metaphase II; (F) anaphase II; (G) telophase II; and (H) tetrad stage. Scale bars $=10 \mu \mathrm{m}$

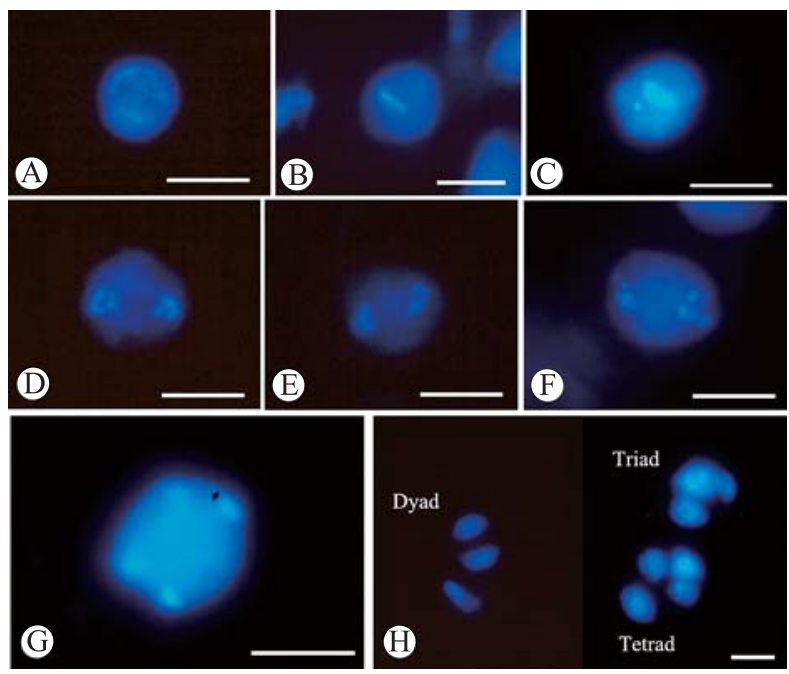

Fig. 5. Meiotic stages of the haploid: (A) prophase I; (B) metaphase I; (C) anaphase I; (D) telophase I and prophase II; (E) metaphase II; (F) anaphase II; (G) telophase II; and (H) tetrad stage. Scale bars $=10 \mu \mathrm{m}$.

(Fig. 5E, F, G). In additional type of abnormal division was observed in some meiocytes (Fig. 6), in which all the univalent chromosomes remained near the equatorial plate without distributing to either side of the poles at anaphase I (Fig. 6A, B). In addition, nine univalents remaining on the equatorial plate showed mitotic division to segregate each set of chromosomes in the direction of opposite poles during the second meiosis (Fig. 6C). Consequently, microspore types from monads to hexads were observed in the tetrad stage of the haploid. The sizes of their microspores were unequal except for the dyads (Fig. 5H). The mechanism responsible for monad, triad, pentad, and hexad formation was not clear from the present study.

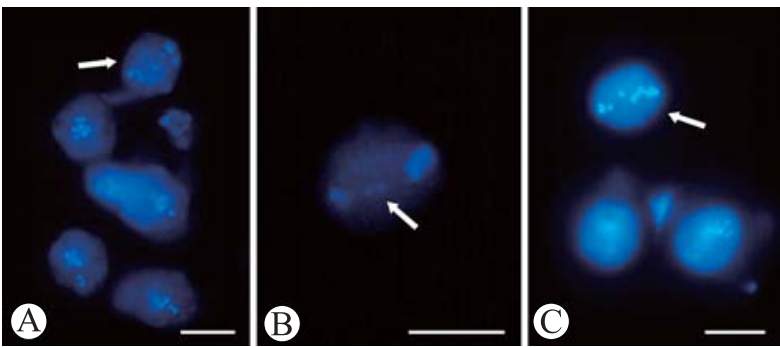

Fig. 6. Abnormalities of the meiotic stage of the haploid. Arrows indicate the abnormal cell: (A) and (B) chromosomes remained in the equatorial plane; and $(\mathrm{C})$ nine bivalents aligned in the equatorial plane. Scale bars $=10 \mu \mathrm{m}$.

At the tetrad stage, the 'Banpeiyu' pummelo predominantly produced normal tetrads (99.2\%) although it had sporads from dyads to pentads (Table 1). In contrast, the haploid had monads, dyads, triads, tetrads, pentads, and hexads, and the frequencies of these microspore types were $1.1 \%, 24.7 \%, 11.9 \%, 61.1 \%$, $1.1 \%$, and $0.1 \%$, respectively. Consequently, the haploid had a high frequency of dyads and triads compared with the 'Banpeiyu' pummelo. We evaluated the fertility of the pollen grains based on their stainability with acetocarmine at anthesis. There was a remarkable reduction of pollen fertility in the haploid. The 'Banpeiyu' pummelo showed a $97.5 \%$ stainability rate, whereas that of the haploid was only $14.1 \%$. The fertility of the pollen grains in the present study $(14.1 \%)$ was higher than that of the previous study (1.6\%) (Yahata et al., 2005a), probably because the tree vigor of the pummelo haploid in the present study was stable.

Some species, such as Brassica napus, Prunus persica, Lycopersicon esculentum, and Capsicum annuum can form fertile gametes in haploid plants (Hesse, 1971; Pooler and Scorza, 1995; Toyama, 1974; Veilleux, 1985; Yan et al., 2000). For fertile gamete formation in the haploid plant, the complete set of the haploid genome, i.e., all chromosomes in the meiocyte, should migrate to the same pole during meiosis I. The probability of occurrence of such an event in the pummelo haploid is theoretically $(1 / 2)^{9}=0.2 \%$. In the present study, however, the pollen fertility of the haploid was $14.1 \%$, which was higher than the expected fertility rate. Meiotic nuclear restitution has been identified as a casual factor in this phenomenon (Ramanna and Jacobsen, 2003). In the haploid plant of Capsicum annuum, Yan et al. (2000) found laggards in many meiocytes of the first division at meiosis of the PMC, which resulted in FDR at meiosis that led to the restitution of pollen fertility in the haploid. They further reported that the microspores formed by FDR were dyads.

Although two successive divisions occurred in the PMC of the haploid, as occurs in normal meiosis, the following abnormalities were observed in some meiocytes: all the univalent chromosomes remained near the equatorial plate without distributing towards either 
Table 1. Frequency of appearance of microspore types in the tetrad stage and pollen fertility in the haploid and the 'Banpeiyu' pummelo.

\begin{tabular}{lccccccc}
\hline & \multicolumn{3}{c}{ No. of microspore type } & \multicolumn{2}{c}{ Pollen stainability } \\
\cline { 2 - 6 } & Monad & Dyad & Triad & Tetrad & Pentad & Hexad & $(\%)$ \\
\hline The haploid & $25(1.1 \%)$ & $569(24.7 \%)$ & $274(11.9 \%)$ & $1409(61.1 \%)$ & $26(1.1 \%)$ & $2(0.1 \%)$ & 14.1 \\
'Banpeiyu' pummelo & $0(0 \%)$ & $1(0.1 \%)$ & $9(0.5 \%)$ & $1749(99.2 \%)$ & $4(0.2 \%)$ & $0(0 \%)$ & 97.5 \\
\hline
\end{tabular}

side of the poles at anaphase I, and nine univalents on the equatorial plate showed normal mitotic division to segregate each set of chromosomes in the direction of opposite poles during the second meiosis. At the tetrad stage, furthermore, numerous dyads were formed. When dyads were assumed to develop into fertile pollen and the pollen grains of the number for each microspore types were formed, the frequency of fertile pollen grains was estimated to be $14.7 \%[(2 \times 569 / 1 \times 25+2 \times 569+3$ $\times 274+4 \times 1409+5 \times 26+6 \times 2) \times 100]$, which is almost equal to the frequency of stainable pollen with acetocarmine $(14.1 \%)$ at anthesis. These results indicate that fertile pollen grains in the haploid were of dyad derivation, as previously reported in the haploid plant of Capsicum annuum. Since the dyads were formed through the arrest of first meiotic division, it can be considered that meiotic nuclear restitution such as FDR took place in the haploid used in the present study.

In conclusion, we investigated the process of meiosis in both gametes in a pummelo haploid and revealed that the lack of EMC formation was responsible for the complete sterility of the female gamete and that unreduced gamete formation by FDR caused partial fertility of the male gamete.

\section{Acknowledgments}

The authors are grateful to Dr. Masahiro Mii of the Faculty of Horticulture, Chiba University, for his advice and critical reading of this manuscript. The authors thank Mr. Kiyoto Harusaki for kindly providing the experimental materials.

\section{Literature Cited}

Aleza, P., J. Juárez, M. Hernández, J. A. Pina, P. Ollitrault and L. Navarro. 2009. Recovery and characterization of a Citrus clementina Hort. ex Tan. 'Clemenules' haploid plant selected to establish the reference whole Citrus genome sequence. BMC Plant Biol. 9: 110.

Bacchi, O. 1943. Cytological observations in Citrus: III. Megasporogenesis, fertilization and polyembryony. Bot. Gaz. 105: 221-225.

Froelicher, Y., J. Bassene, E. Jedidi-Neji, D. Dambier, R. Morillon1, G. Bernardini, G. Costantino and P. Ollitrault. 2007. Induced parthenogenesis in mandarin for haploid production: induction procedures and genetic analysis of plantlets. Plant Cell Rep. 26: 937-944.

Germanà, M. A. 2009. Haploids and doubled haploids in fruit trees. p. 241-263. In: A. Touraev, B. P. Forster and S. M. Jain (eds.). Advances in haploid production in higher plants. Springer Netherlands, Dordrecht.
Germanà, M. A. and B. Chiancone. 2001. Gynogenetic haploids of Citrus after in vitro pollination with triploid pollen grains. Plant Cell Tissue Organ Cult. 66: 59-66.

Germanà, M. A. and B. Chiancone. 2003. Improvement of Citrus clementina Hort. ex Tan. microspore-derived embryoid induction and regeneration. Plant Cell Rep. 22: 181-187.

Germanà, M. A., Y. Y. Wang, M. G. Barbagallo, G. Iannolino and F. G. Crescimanno. 1994. Recovery of haploid and diploid plantlets from anther culture of Citrus clementina Hort. ex Tan. and Citrus reticulata Blanco. J. Hort. Sci. 69: 473-480.

Hesse, C. O. 1971. Monoploid peaches, Prunus persica Batsch: description and meiotic analysis. J. Amer. Soc. Hort. Sci. 96: 326-330.

Hidaka, T., Y. Yamada and T. Shichijo. 1979. In vitro differentiation of haploid plants by anther culture in Poncirus trifoliata (L.) Raf. Japan. J. Breed. 29: 248-254.

Iwamasa, M. 1976. Cultivars of Citrus, p. 9-18. Shizuoka Citrus Grower's Coop. Assoc. Shimizu (In Japanese).

Oiyama, I. and S. Kobayashi. 1993. Haploids obtained from diploid $\times$ triploid cross of Citrus. J. Japan. Soc. Hort. Sci. 62: 89-93.

Pooler, M. and R. Scorza. 1995. Occurrence of viable eggs in haploid peach. Fruit Var. J. 49: 239-241.

Ramanna, M. S. and E. Jacobsen. 2003. Relevance of polyploidization for crop improvement-A review. Euphytica 133: 3-18.

Toolapong, P., H. Komatsu and M. Iwamasa. 1996. Triploids and haploid progenies derived from small seeds of 'Banpeiyu' pummelo, crossed with 'Ruby Red' grapefruit. J. Japan. Soc. Hort. Sci. 65: 255-260.

Toyama, T. K. 1974. Haploidy in peach. HortScience 9: 187-188.

Veilleux, R. 1985. Diploid and polyploid gametes in crop plants: Mechanisms of formation and utilization in plant breeding. Plant Breed. Rev. 3: 253-288.

Yahata, M., S. Harusaki, H. Komatsu, K. Takami, H. Kunitake, T. Yabuya, K. Yamashita and P. Toolapong. 2005a. Morphological characterization and molecular verification of a fertile haploid pummelo (Citrus grandis Osbeck). J. Amer. Soc. Hort. Sci. 130: 34-40.

Yahata, M., H. Kurogi, H. Kunitake, K. Nagano, T. Yabuya, K. Yamashita and H. Komatsu. 2005b. Evaluation of reproduction in a haploid pummelo by crossing with several diploid citrus cultivars. J. Japan. Soc. Hort. Sci. 74: 281-288.

Yahata, M., K. Yasuda, K. Nagasawa, S. Harusaki, H. Komatsu and H. Kunitake. 2010. Production of haploid plant of 'Banpeiyu' pummelo [Citrus maxima (Burm.) Merr.] by pollination with soft X-ray irradiated pollen. J. Japan. Soc. Hort. Sci. 79: 239-245.

Yamashita, K. and K. Yamaguchi. 1992. Histological and physiological disorders of pollen of tetraploid Hyuganatsu. J. Japan. Soc. Hort. Sci. 60: 845-849 (In Japanese with English abstract).

Yan, L. Y., X. Z. Zhang and G. J. Liu. 2000. Occurrence of unreduced gametes and ploidy restoration in haploid Capsicum annuиm L. J. Hort. Sci. Biotechnol. 75: 195-197. 